\title{
Recognition and Imaging of Point Defect Diffusion, Recombination, and Reaction During Growth of Czochralski-Silicon Crystals
}

\author{
MASATAKA HOURAI (D), ${ }^{1,2}$ EIICHI ASAYAMA, ${ }^{1}$ HIDESHI NISHIKAWA, ${ }^{1}$ \\ MANABU NISHIMOTO, ${ }^{1}$ TOSHIAKI ONO ${ }^{1}$ and MASAHIKO OKUI ${ }^{1}$
}

1.-Technology Division, SUMCO Corporation, 1-52 Kubara, Yamashiro-cho, Imari, Saga 8494256, Japan. 2.—e-mail: mhourai@sumcosi.com

\begin{abstract}
The behavior of point defects was visualized in lightly and heavily boron (B)doped Czochralski-silicon (CZ-Si) crystals by employing a special growth technique, namely, rapidly cooling a growing crystal after it is detached from the $\mathrm{Si}$ melt. In the case of crystal growth with a high pulling rate, an anomalous oxygen precipitation (AOP) region dominated by vacancies appeared, whereas in the case of crystal growth with a low pulling rate, a dislocation loop region dominated by self-interstitials appeared. In the crystals cooled rapidly after halting growth for several hours, self-interstitials flowed into the AOP region and dislocation loop regions formed and expanded, while the AOP region shrunk due to diffusion of excess vacancies to the crystal surface and void regions. These transient changes in the point defect distribution were reproduced using a point defect simulator. Defect regions related to self-interstitials could not be confirmed in the heavily B-doped crystals with resistivities of $10 \mathrm{~m} \Omega \mathrm{cm}$ or less, where the void- and oxidation-induced stacking fault (OSF)-ring regions disappeared completely at the center of the crystal. These results show that the behaviors of point defects in heavily doped CZ-Si crystals with various impurities are important research subjects in relation to future advanced power applications.
\end{abstract}

Key words: Czochralski-silicon crystal, point defect, vacancy, self-interstitial, void, dislocation

\section{INTRODUCTION}

Point defects in Czochralski (CZ)-Si crystals form aggregates, such as voids ${ }^{1,2}$ and dislocation clusters, ${ }^{3}$ and secondary defects, such as oxygen precipitates, stacking faults, and/or dislocations due to reactions with oxygen, carbon, or nitrogen. Since these defects directly affect the performance and characteristics of semiconductor devices, it is important to understand and control the behavior of point defects during CZ-Si crystal growth. Harada et al. ${ }^{4}$ and Abe et al. ${ }^{5}$ imaged point defect behavior during crystal growth by using special experimental techniques. Such means can reveal, directly or indirectly, the spatial and dynamic distribution of point defects in growing CZ-Si crystals at high temperatures just after solidification and formation of point defect aggregates. In this report, we review several findings obtained from our experiments that used similar growth techniques on lightly and heavily boron (B)-doped CZ-Si crystals grown with high and low pulling rates. 


\section{BEHAVIOR OF POINT DEFECTS IN LIGHTLY DOPED CZ-SI CRYSTALS}

\section{Rapid Cooling After Crystal Detachment from Si Melt}

Harada et al. ${ }^{4}$ and Abe et al. ${ }^{5}$ proposed rapid cooling of the crystal after detaching it from the Si melt as a powerful tool to study point defect behavior during CZ-Si crystal growth. For example, they used this technique to grow two crystals with high and low pulling rates. In this experiment, longitudinal wafers cut parallel to the growth axis of the crystals were subjected to a heat treatment for oxygen precipitation and were observed by X-ray topography. Anomalous oxygen precipitation $(\mathrm{AOP})^{4,5}$ was found just above the detached solidliquid interface of the rapidly cooled crystals. In this AOP region, excess vacancies formed complexes with themselves or with oxygen atoms during rapid cooling, and these complexes acted as nuclei to enhance oxygen precipitation in the longitudinal wafers during the heat treatments applied later. In the crystal grown with the high pulling rate, AOP spread over a wide area, but in the crystal grown with the low pulling rate, the AOP region shrank remarkably in the vicinity of the detached interface. These results indicated that the growth technique is very useful for visualizing point defect behavior during crystal growth.

In a similar way as described above, we grew a 100-mm-diameter lightly B-doped CZ-Si crystal at a pulling rate of $1.0 \mathrm{~mm} / \mathrm{min}$ until it reached $400 \mathrm{~mm}$ in length, at which point it was detached from the $\mathrm{Si}$ melt and cooled rapidly. ${ }^{6}$ AOP was observed in the area at temperatures higher than about $1100^{\circ} \mathrm{C}$ during growth above the solid-liquid interface. Void defects were measured at the center of the crystal along the growth axis, and it was found that they appeared only at temperatures below $1100^{\circ} \mathrm{C}$. This meant that the AOP terminated at about $1100^{\circ} \mathrm{C}$ because the vacancy super-saturation dropped sharply due to the aggregation of vacancies to form voids.

A similar experiment was conducted by Okui et al. ${ }^{7,8}$ Figure 1 shows schematic drawings of defect regions observed in a longitudinal section of 150 mm-diameter lightly B-doped CZ-Si crystals that were grown at pulling rates of $1.0,0.7$ and $0.35 \mathrm{~mm} /$ min, and cooled rapidly after detaching from the $\mathrm{Si}$ melt. In the case of the high pulling rate, $1.0 \mathrm{~mm} /$ min (Fig. 1a), the AOP terminated at about $1110^{\circ} \mathrm{C}$, a similar result to that mentioned above. However, at the medium pulling rate, $0.7 \mathrm{~mm} / \mathrm{min}$ (Fig. $1 \mathrm{~b}$ ), the AOP region shrank to the central area and an oxidation-induced stacking fault (OSF)-ring region appeared next to the outer boundary region of the AOP. In addition, the AOP termination temperature fell to about $1060^{\circ} \mathrm{C}$. From this, it was concluded that lowering the pulling rate reduces the vacancy concentration so that the AOP shrinks to the central area and the void formation temperature decreases.
In the case of the low pulling rate, $0.35 \mathrm{~mm} / \mathrm{min}$ (Fig. 1c), the AOP region shrank remarkably near the solid-liquid interface, which is similar to what was reported by Abe et al. ${ }^{5}$ However, an etch-pit observation after Secco's preferential etching of the as-grown longitudinal wafers also found that dislocation clusters formed with a density of $10^{4}-$ $10^{5} \mathrm{~cm}^{-3}$ around $1000^{\circ} \mathrm{C}$. In addition, Okui found that, ${ }^{7}$ as shown in Fig. 1c, after $\mathrm{Cu}$ decoration of the crystal grown at $0.35 \mathrm{~mm} / \mathrm{min}$, a wide $\mathrm{Cu}$-decorated area appeared between the AOP and the dislocation cluster region, and a narrow defect-free region appeared between the AOP and Cu-decorated area. Transmission electron microscopy (TEM) of the $\mathrm{Cu}$ decorated area revealed a high concentration of hexagonal and petal-like micro dislocation loops on (111) planes, $10^{9}-10^{10} \mathrm{~cm}^{-3}$. A detailed TEM analysis identified them to be interstitial type. From these observations, we propose that the point defect behavior in the slow-growing crystal is as follows: vacancies are predominant near the solid-liquid interface, but they are replaced by self-interstitials because they diffuse faster than vacancies, and a narrow defect-free region forms next to the AOP. The excess self-interstitials spread throughout the $\mathrm{Cu}$-decorated area and form dislocation clusters at about $1000^{\circ} \mathrm{C}$, decreasing the super-saturation of self-interstitials.

We tried to reproduce the growth experiments performed by Okui, shown in Fig. $1 \mathrm{a}, \mathrm{b},{ }^{8}$ and $c,{ }^{7}$ by using a point defect simulator ${ }^{9,10}$ based on the Voronkov model. ${ }^{11}$ This model describes the diffusion and pair recombination reaction of vacancies and self-interstitials. (The point defect simulation is described in the "Appendix".) The simulator was used to calculate the two-dimensional distributions of the point defect concentrations. In the following, to discuss the behavior of point defects observed in our experiments, we will use one-dimensional point defect distributions calculated along with the crystal growth axis.

Figure 2 shows the concentration distributions of point defects along the crystal growth axis as a function of temperature in the growing crystals above the solid-liquid interface just before detachment. Regarding the physical properties of point defects, it is considered that the thermal equilibrium concentration is larger for vacancies than for self-interstitials, which diffuse faster than vacancies. In addition, since thermal equilibrium is assumed to be maintained in the point defect concentration at the solid-liquid interface and crystal surface, vacancies become dominant at temperatures near the melting point regardless of the crystal pulling rate. This can be seen in the plots of Fig. 2a, b, and c, which were calculated for crystals grown with different pulling rates.

In the case of fast pulling $(1.0 \mathrm{~mm} / \mathrm{min})$, vacancies become dominant because they are carried to the low-temperature area by the fast crystal growth and the self-interstitials, which are lower in 


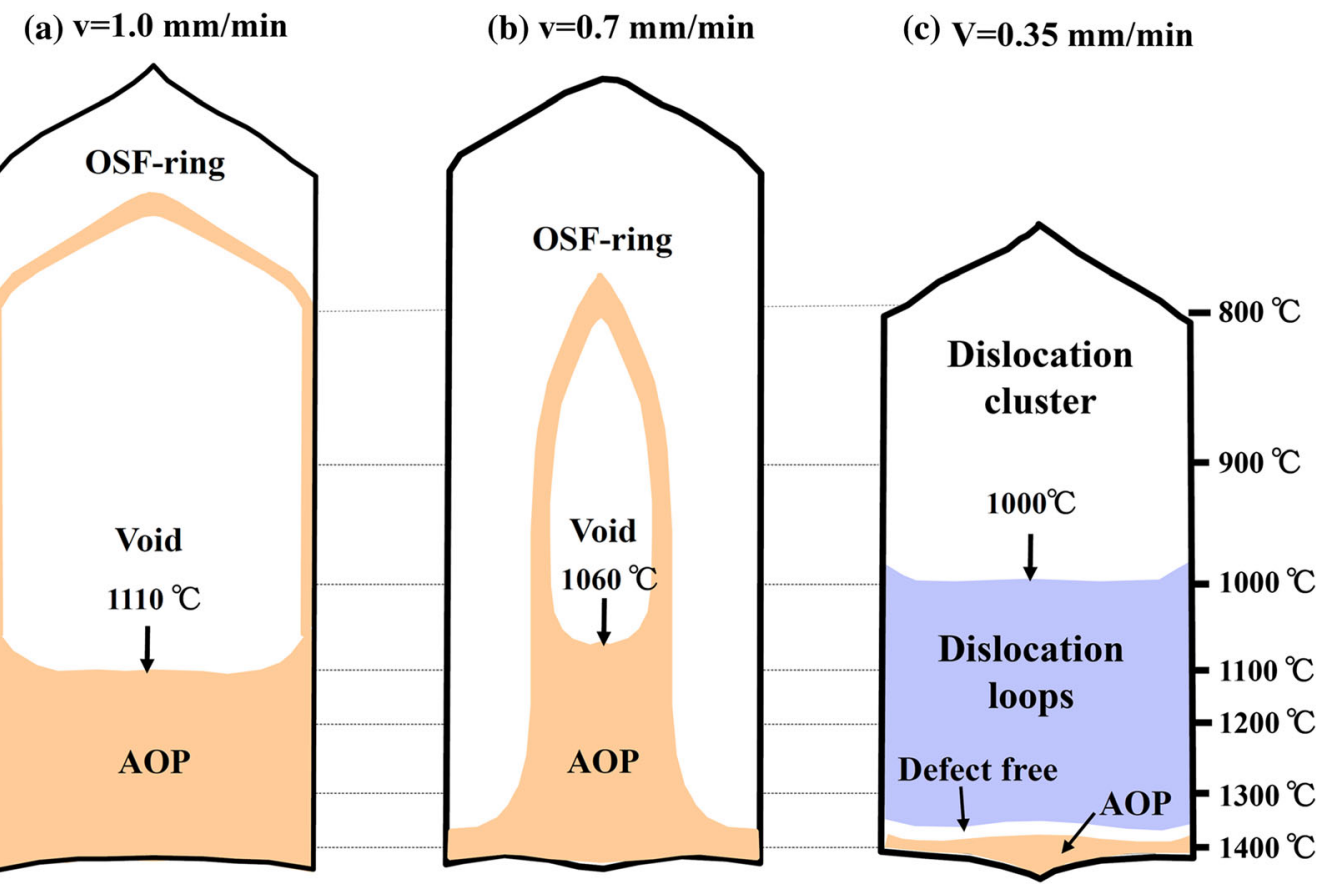

Fig. 1. Schematic drawings of defect regions due to point defects observed in lightly B-doped CZ-Si crystals grown at different pulling rates, (a) $1.0 \mathrm{~mm} / \mathrm{min}$, (b) $0.7 \mathrm{~mm} / \mathrm{min}$, and (c) $0.35 \mathrm{~mm} / \mathrm{min}$, and cooled rapidly after detaching from the Si melt.

(a) $\mathbf{v}=\mathbf{1 . 0} \mathrm{mm} / \mathrm{min}$

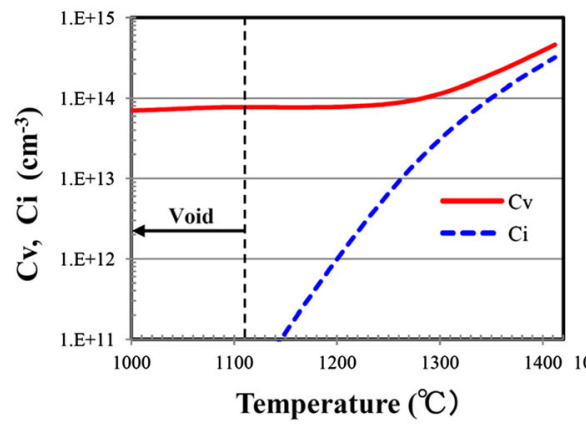

(b) $\mathrm{v}=\mathbf{0 . 7} \mathrm{mm} / \mathrm{min}$

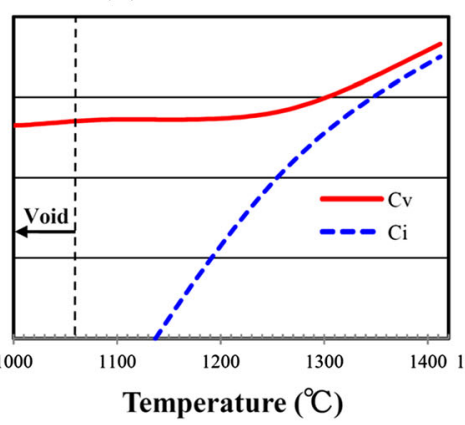

(c) $\mathrm{v}=0.35 \mathrm{~mm} / \mathrm{min}$

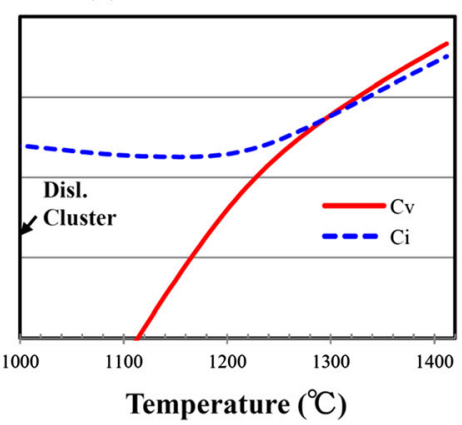

Fig. 2. Point defect concentrations in crystals growing with different puling rates, (a) $1.0 \mathrm{~mm} / \mathrm{min}$, (b) $0.7 \mathrm{~mm} / \mathrm{min}$, and (c) $0.35 \mathrm{~mm} / \mathrm{min}$, just before detachment from Si melt as shown in Fig. 1, which were calculated by a point defect simulator using the point defect parameters optimized by Nishimoto et al. ${ }^{9,10} \mathrm{Cv}$ and $\mathrm{Ci}$ are the concentrations of vacancies and self-interstitials, respectively.

concentration, are annihilated by recombination with vacancies (Fig. 2a). On the other hand, in the case of slow pulling $(0.35 \mathrm{~mm} / \mathrm{min})$, self-interstitials outnumber vacancies because they diffuse faster into the low-temperature area. Therefore, after some of them recombine with vacancies, the remaining self-interstitials become the dominant point defects except in the vicinity of the solid-liquid interface (Fig. 2c). In the case of the medium pulling rate $(0.7 \mathrm{~mm} / \mathrm{min})$, the vacancy concentration is lower than in the case of pulling at $1.0 \mathrm{~mm} / \mathrm{min}$ (Fig. 2b). Since this simulation does not include agglomeration reactions of voids and dislocation clusters, the point defect concentrations are maintained even below $1100^{\circ} \mathrm{C}$ for voids and about $1000^{\circ} \mathrm{C}$ for dislocation clusters.

\section{Crystal Growth Halting}

Next, we will review other findings obtained from an experiment using growth halting and rapid cooling after detachment. Through this operation, defect reactions, which occur during the cooling process of the crystal growth, can be promoted. Nishikawa et al. ${ }^{12}$ grew two $150-\mathrm{mm}$-diameter crystals at a pulling rate of $1.0 \mathrm{~mm} / \mathrm{min}$. One was directly detached and cooled rapidly; the other was growth-halted for $5 \mathrm{~h}$ before detaching and cooled rapidly. Figure 3a shows the void density observed by an optical precipitate profiler $(\mathrm{OPP})^{13}$ as a function of temperature just before detaching. The distributions of voids in the crystals are almost identical. Figure $3 \mathrm{~b}$ shows the OPP signal size of voids, which is proportional to the cube of the defect radius. The void sizes in the crystal for which 
(a)

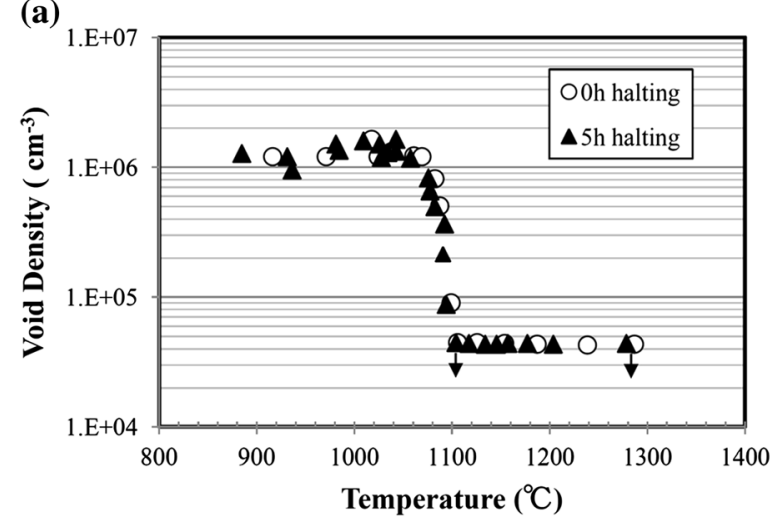

(b)

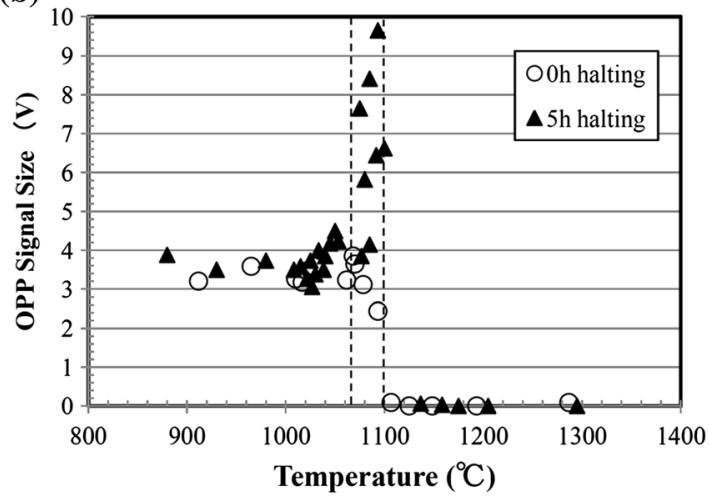

Fig. 3. Distributions of (a) void density and (b) signal size observed by OPP as a function of crystal temperature just before rapid cooling after halting growth for 0 and $5 \mathrm{~h}$. Arrows show detection limits. Adapted from. ${ }^{12}$ Copyright 1997, The Japan Society of Applied Physics.

(a) 1h halting

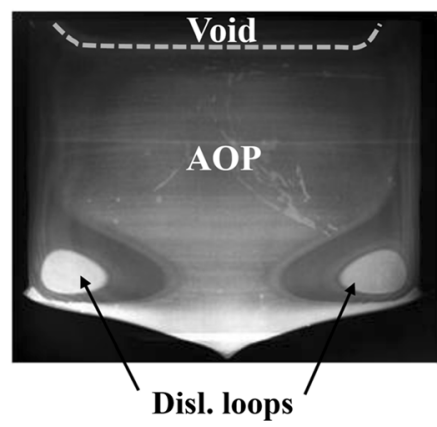

(b) 3h halting

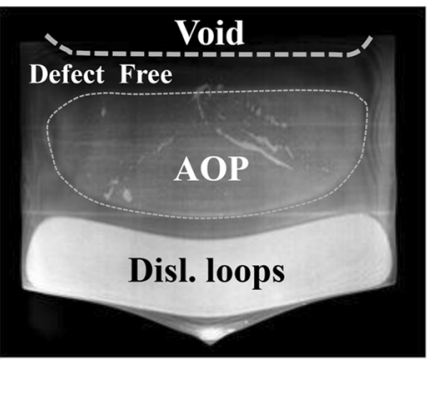

(c) 5h halting

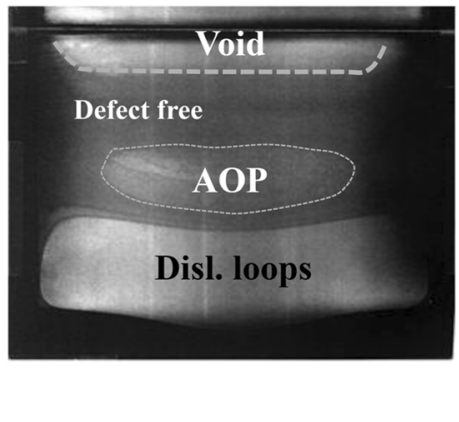

Fig. 4. X-ray topography images after $\mathrm{Cu}$ decoration showing defect distributions due to point defects in crystals grown at $1.0 \mathrm{~mm} / \mathrm{min}$ and cooled rapidly (a) $1 \mathrm{~h}$, (b) $3 \mathrm{~h}$, and (c) $5 \mathrm{~h}$ after halting growth.

growth was halted for $5 \mathrm{~h}$ increased significantly from $1100^{\circ} \mathrm{C}$ to $1070^{\circ} \mathrm{C}$. Accordingly, it can be concluded that voids formed only in a very narrow temperature range, i.e., within $30^{\circ} \mathrm{C}$, below $1100^{\circ} \mathrm{C}$.

In addition, Nishikawa et al. ${ }^{12}$ observed the shapes of crystal-originated particles or pits $(\mathrm{COP})^{14}$ on the surface of mirror-polished wafers cut from both crystals by using an atomic force microscope (AFM) and compared them with those after the wafers had been immersed in HF. It was found that the inner walls of the voids were covered with oxide film which was significantly thicker for COPs in the 5-h growth-halted crystal than in the crystal that was not growth halted. In addition, the oxide film thickness in the growth-halted crystal was dependent on the holding temperature, from $1100^{\circ} \mathrm{C}$ to $900^{\circ} \mathrm{C}$, during growth halting. These findings indicated that void formation is stopped by the growth of oxide film on the inner wall of the void and that oxide film growth continues in a relatively wide temperature range down to about $900^{\circ} \mathrm{C}$.

To observe the behavior of point defects in crystals during growth halting, another three 150mm-diameter lightly B-doped CZ-Si crystals were pulled to $400 \mathrm{~mm}$ in length at a rate of $1.0 \mathrm{~mm} / \mathrm{min}$ and then were growth halted for $1 \mathrm{~h}, 3 \mathrm{~h}$, and $5 \mathrm{~h}$ before detachment and rapid cooling. Figure 4 shows X-ray topography images of the crystals after $\mathrm{Cu}$ decoration. After $1 \mathrm{~h}$ of growth halting, an island-like self-interstitial-dominated dislocation loop region appeared in the outer periphery of the AOP region (Fig. 4a). After $3 \mathrm{~h}$ of growth halting, the dislocation loop region expanded above the solid-liquid interface, attaining a width of about $30 \mathrm{~mm}$ (Fig. 4b). In addition, the AOP region shrank and took on an island shape above the dislocation loop region, and a narrow defect-free region appeared around the AOP region. After $5 \mathrm{~h}$ of halting, the dislocation loop region further expanded to a width of about $40 \mathrm{~mm}$, while the island-like AOP region further shrank and the defect-free region expanded (Fig. 4c).

Figure 5 shows the changes in the point defect distributions at the centers of the crystals during growth halting, as calculated by the point defect simulator. After $1 \mathrm{~h}$ of growth halting, there was a decrease in the vacancy concentration in the vicinity of the solid-liquid interface, but at this point, the vacancies still dominated. After $3 \mathrm{~h}$ of growth halting, a self-interstitial dominant region appeared, and it had expanded after $5 \mathrm{~h}$ of growth halting. During crystal growth, the solid-liquid 
interface and crystal surface are assumed to be in thermal equilibrium. However, since the interior of the crystal is not in an equilibrium state, the state of the point defects changes transiently toward thermal equilibrium during growth halting. As shown in Fig. 2a, just before growth halting, there are excess vacancies in the AOP region above the solid-liquid interface, while self-interstitials are depleted. Therefore, the excess vacancies diffuse out toward the crystal surface and disappear there. It can also be inferred that excess vacancies diffuse into the void region and enhance void growth there. On the other hand, self-interstitials flow from the solidliquid interface into the AOP region to recover from their depleted state, and they transiently outnumber vacancies. Through mutual recombination, the self-interstitial dominated region remains in the AOP region, as in the case of the island region, and expands during growth halting, as shown in Fig. 5.

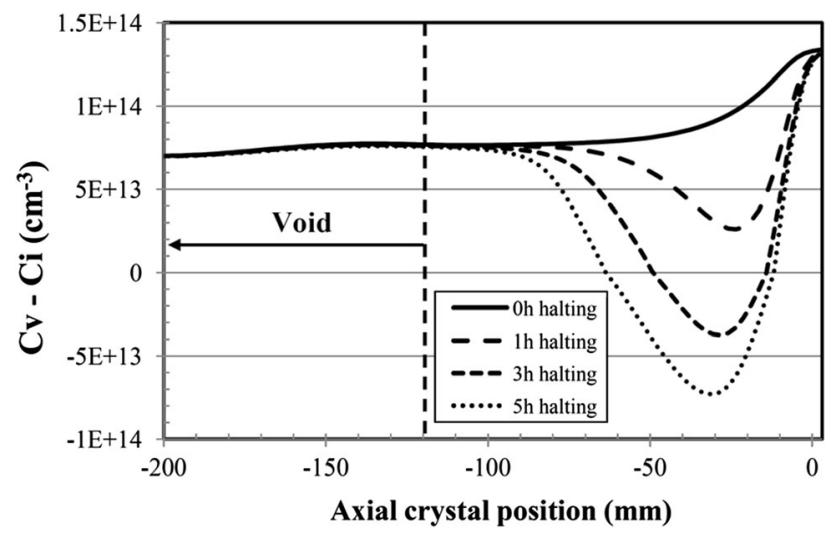

Fig. 5. Distributions of concentration difference between vacancies and self-interstitials in growing crystals just before detachment, after halting growth for $0,1,3$ and $5 \mathrm{~h}$ as shown in Fig. 4, which were calculated by a point defect simulator using the point defect parameters optimized by Nishimoto et al. ${ }^{9,10}$.

\section{BEHAVIOR OF POINT DEFECTS IN HEAVILY B-DOPED CZ-SI CRYSTALS}

In this section, we describe the defect regions that Asayama et al. first observed in heavily B-doped crystals. ${ }^{15}$ Figure 6 a shows the diameter of the void region, i.e., the OSF-ring region in the heavily $\mathrm{B}$ doped crystals as functions of B concentration and resistivity. In this figure, the triangles are the data of Suhren et al., ${ }^{16}$ and the solid black circles and squares are our results. The figure shows that the void and OSF-ring regions began to shrink from about $20 \mathrm{~m} \Omega \mathrm{cm}$ and disappeared completely at the crystal center when the resistivity was below $10 \mathrm{~m} \Omega \mathrm{cm}$. As shown in Fig. $6 \mathrm{~b},{ }^{17,18}$ this phenomenon is very similar to what occurred in lightly B-doped crystals with decreasing $v / G$, where $v$ is the crystal growth rate and $G$ is the axial temperature gradient in the crystal near the melting point. Using CZ furnaces with four different temperature gradients $G$, we grew thirteen 150-mm-diameter crystals at three different pulling rates and found that the OSF-ring radii depend on the temperature gradient $G$ as well as on the pulling rate v. We plotted the data against $v / G$ and found that they were normalized by $v / G$ becoming zero at a certain critical value. When $v / G$ is larger than the critical value, vacancies become the dominant point defects and voids appear inside the OSF-ring. When $v / G$ is smaller than the critical value, self-interstitials become dominant and dislocation clusters appear outside the OSFring. From the similarity in the behaviors of the voids and OSF-ring regions, it has not been questioned that heavily B-doped crystals with resistivities less than $10 \mathrm{~m} \Omega \mathrm{cm}$ are dominated by selfinterstitials. However, we could not observe dislocation clusters in such crystals, from which the void and OSF-ring regions disappeared completely. Here, Asayama wondered whether such heavily Bdoped crystal with resistivities less than $10 \mathrm{~m} \Omega \mathrm{cm}$ are actually interstitial type or not and decided to
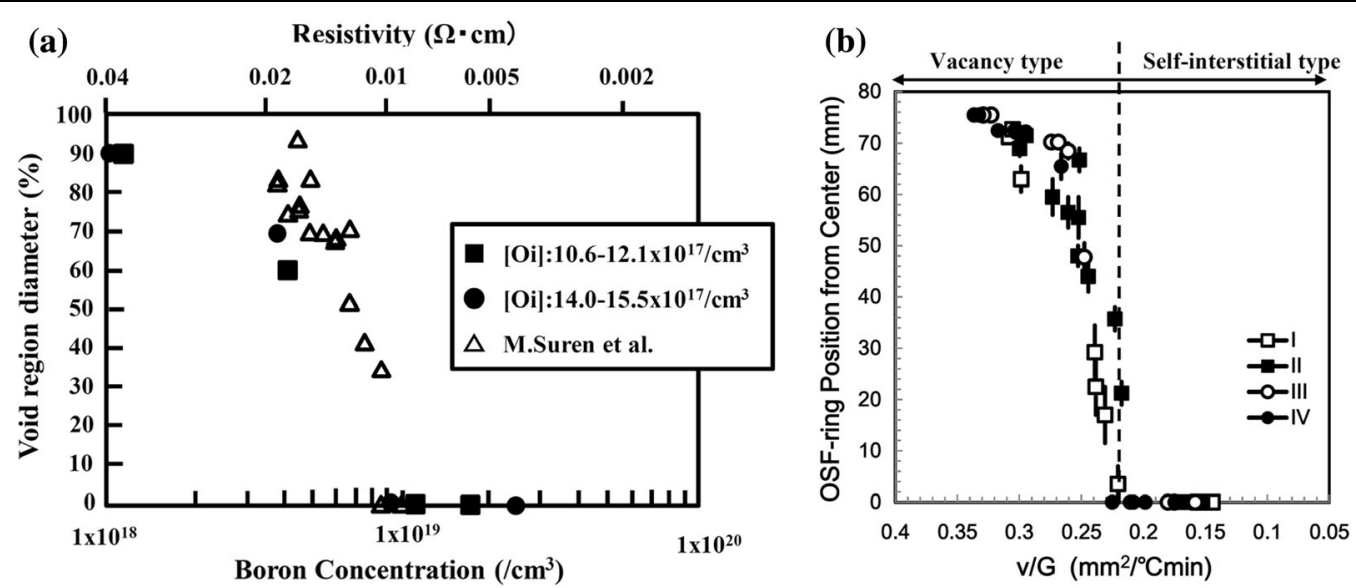

Fig. 6. (a) Correlation between void region diameter and boron concentration. Adapted with permission. ${ }^{15}$ Copyright 1999 , The Japanese Association for Crystal Growth, (b) OSF-ring position from center as a function v/G. Adapted with permission. ${ }^{17}$ Copyright 1995 , Trans Tech Publications Ltd. 
study the behavior of point defects in them by using the rapid cooling technique.

Figure 7 shows X-ray topography images of 150mm-diameter heavily B-doped crystals that were grown at a high pulling rate, $1.0 \mathrm{~mm} / \mathrm{min}$, and cooled rapidly after detaching from the Si melt. In the case of the crystal with a resistivity of $18 \mathrm{~m} \Omega \mathrm{cm}$, AOP and OSF rings shrank in a similar way as in the lightly B-doped crystal grown at the medium pulling rate shown in Fig. 1b. In contrast, no defect regions were found in the $9 \mathrm{~m} \Omega \mathrm{cm}$ crystal. Asayama grew three heavily B-doped crystals with different B concentrations at a low pulling rate of $0.4 \mathrm{~mm} / \mathrm{min}$ and cooled them rapidly. Figure 8 shows the X-ray topography images after $\mathrm{Cu}$ decoration. AOP, defect-free, micro-dislocation loops, and dislocation cluster regions were clearly observed in the crystal with a resistivity of $40 \mathrm{~m} \Omega \mathrm{cm}$ (Fig. 8a), which is very similar to the case of lightly B-doped crystal grown at a low pulling rate (Fig. 1c). Accordingly, it can be concluded that the defect regions related to the point defects in lightly B-doped crystals behave in the same way down to at least $40 \mathrm{~m} \Omega \mathrm{cm}$. On the other hand, the crystal with $18 \mathrm{~m} \Omega \mathrm{cm}$ showed a contrast change in the dislocation cluster region at temperatures below $900^{\circ} \mathrm{C}$. However, there were no other defect regions such as micro dislocation loops or AOP in the higher temperatures. Moreover, hardly any defect regions were observed in the crystal with $9 \mathrm{~m} \Omega \mathrm{cm}$. Asayama then applied twostep heat treatments for oxygen precipitation to the crystals with resistivities of $18 \mathrm{~m} \Omega \mathrm{cm}$ and $9 \mathrm{~m} \Omega \mathrm{cm}$. In this case, an AOP region appeared in the vicinity of the solid-liquid interface of the $18 \mathrm{~m} \Omega \mathrm{cm}$ crystal, but not in the $9 \mathrm{~m} \Omega \mathrm{cm}$ crystal. Thus, we cannot judge yet whether heavily B-doped crystals with resistivities less than $10 \mathrm{~m} \Omega \mathrm{cm}$ are actually selfinterstitial dominated or not.

Here, we will briefly review the previous experimental and theoretical studies on heavily doped CZ-Si crystals with B and other impurities. de Kock et al. $^{19}$ observed interstitial dislocation loops in

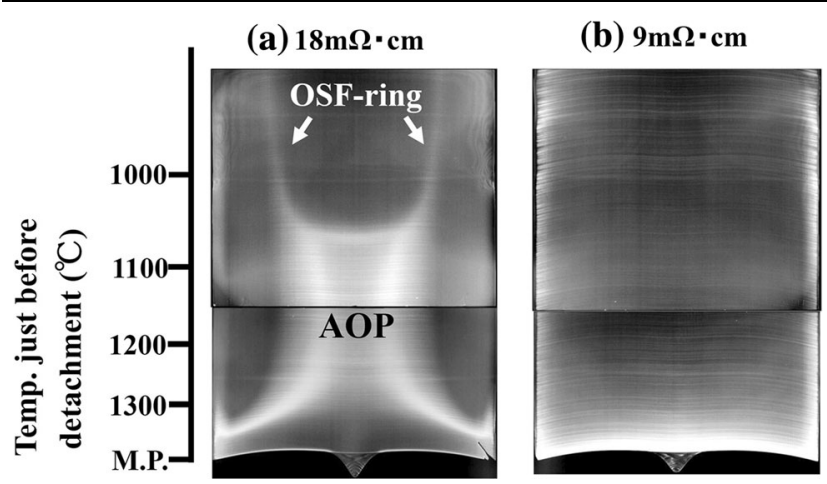

Fig. 7. X-ray topography images for heavily B-doped Si crystals formed with a pulling rate of $1.0 \mathrm{~mm} / \mathrm{min}$ followed by detachment from Si melt, with resistivities of (a) $18 \mathrm{~m} \Omega \mathrm{cm}$ and (b) $9 \mathrm{~m} \Omega \mathrm{cm}$. The samples were subjected to $800^{\circ} \mathrm{C} / 4 \mathrm{~h}+1000^{\circ} \mathrm{C} / 16 \mathrm{~h}$. Adapted with permission. ${ }^{15}$ Copyright 1999, The Japanese Association for Crystal Growth. small-diameter CZ-Si crystals that were heavily Bdoped to a concentration of $3 \times 10^{18} \mathrm{~cm}^{-3}$ (converted resistivity ${ }^{20}: 21 \mathrm{~m} \Omega \mathrm{cm}$ ) and grown with a pulling rate of $1.0 \mathrm{~mm} / \mathrm{min}$. In their experiment, the pulling rate could be varied from $0.6 \mathrm{~mm} / \mathrm{min}$ to $4 \mathrm{~mm} / \mathrm{min}$, so the crystals were considered to have been pulled slowly. Therefore, the observed dislocation loops are similar to the dislocation clusters observed in the low-speed pulled crystal with a resistivity of $18 \mathrm{~m} \Omega \mathrm{cm}$ (Fig. $8 \mathrm{~b}$ ). On the other hand, Dornberger et al. ${ }^{21}$ grew many heavily B-doped CZSi crystals with diameters of 125,150 , and $200 \mathrm{~mm}$ by using high-speed pulling. They inferred that the heavily B-doped crystals with resistivities below $7 \mathrm{~m} \Omega \mathrm{cm}$ were interstitial dominated, because the void and OSF-ring regions disappeared at the crystal center. However, below $10 \mathrm{~m} \Omega \mathrm{cm}$, neither vacancy- nor interstitial-related defects were observed in crystals doped with up to $2 \times 10^{19} \mathrm{~cm}^{-3}$ of $\mathrm{B}(5 \mathrm{~m} \Omega \mathrm{cm}) .{ }^{16,22}$

Sueoka et al. ${ }^{23}$ studied the effects of various impurities on point defect concentrations in $\mathrm{Si}$ crystals by using a first-principle calculation and showed that as the concentrations of $\mathrm{B}$ and $\mathrm{C}$ increase, the dominant point defects change from vacancies to self-interstitials. In particular, for B concentrations from $2 \times 10^{18}$ atoms $/ \mathrm{cm}^{3}$ to $8 \times 10^{18}$ atoms $/ \mathrm{cm}^{3}$ (converted resistivity: 30 to $10 \mathrm{~m} \Omega \mathrm{cm}$ ), the B concentration dependence of the calculated critical $v / G$ normalized by the intrinsic value agrees well with Nakamura's experimental results ${ }^{24}$ concerning the shrinkage of the OSF-ring and void region in crystals doped with $B$ in different concentrations. In addition, they predicted that selfinterstitials would become dominant in crystals

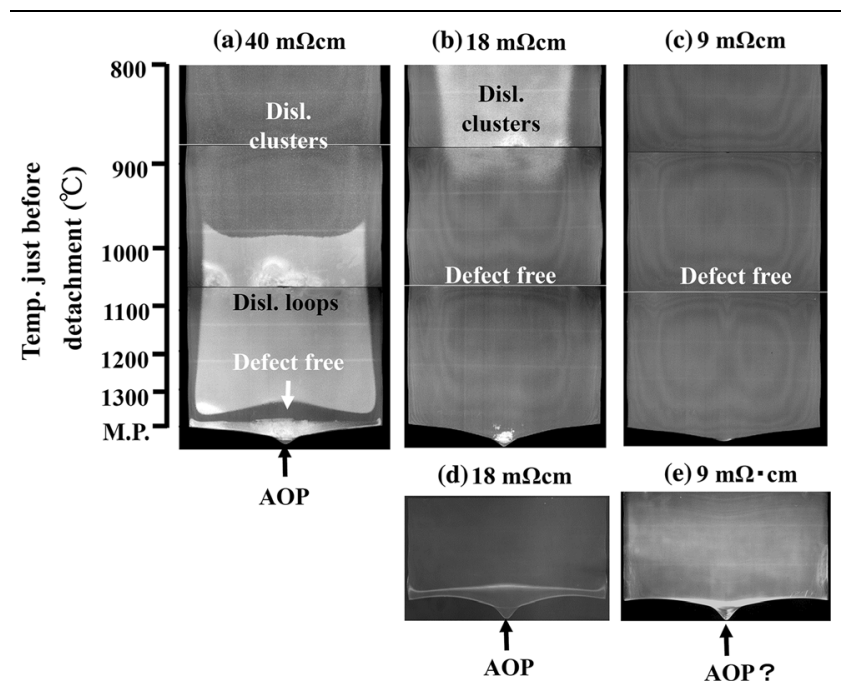

Fig. 8. X-ray topography images of heavily B-doped CZ-Si crystals formed at a pulling rate of $0.4 \mathrm{~mm} / \mathrm{min}$ followed by detachment from Si melt, with resistivities of (a) $40 \mathrm{~m} \Omega \mathrm{cm}$, (b) $18 \mathrm{~m} \Omega \mathrm{cm}$, (c) $9 \mathrm{~m} \Omega \mathrm{cm}$ : Cu decoration, (d) $18 \mathrm{~m} \Omega \mathrm{cm}$, and (e) $9 \mathrm{~m} \Omega \mathrm{cm}: 800^{\circ} \mathrm{C} / 4 \mathrm{~h}+1000^{\circ} \mathrm{C} /$ $16 \mathrm{~h}$. Adapted with permission. ${ }^{15}$ Copyright 1999, The Japanese Association for Crystal Growth. 
doped with B concentrations higher than $2 \times 10^{19} \mathrm{~cm}^{-3}$ (converted resistivity less than $5 \mathrm{~m} \Omega \mathrm{cm}$ ). However, to the best of the author's knowledge, no experimental studies have been reported on crystals doped with such high B concentrations, so this theoretical prediction remains to be confirmed experimentally.

Sueoka et al. also showed that the concentration of vacancies in Si crystals doped with $n$-type impurities such as $\mathrm{P}, \mathrm{As}, \mathrm{Sb}$ and $\mathrm{Bi}$ increases with increasing concentration of $n$-type impurities. However, Voronkov et al. $^{25}$ argued that although the vacancy concentration increases with increasing concentration of $\mathrm{As}$ and $\mathrm{P}$, it becomes zero or neutral after a peak around an impurity concentration of $1 \times 10^{19} \mathrm{~cm}^{-3}$ due to an interaction between the dopant impurities and point defects. This is clearly different from the theoretical prediction of Sueoka et al. Why does this discrepancy occur? It could be due to a lack of reliable experimental information concerning point defects in Si crystals doped with various impurities close to the solubility limits.

Now let us briefly review the recent state of experimental and theoretical investigations on the behavior of defects in n-type crystals that have been heavily doped with As or P. It is known ${ }^{26-28}$ that the void density in such crystals increases with increasing concentration of As or P, but voids suddenly disappear when the concentration exceeds a certain critical value $\left(2-3 \times 10^{19}\right.$ atoms $\left./ \mathrm{cm}^{3}\right)$. In particular, Senda et al. ${ }^{29}$ recently reported that plate-like defects with an average diameter of about $100 \mathrm{~nm}$ and maximum diameter of about $200 \mathrm{~nm}$ formed in a red phosphorus-doped crystal with $8 \times 10^{19}$ atoms $/ \mathrm{cm}^{3}$ and a resistivity of $0.87 \mathrm{~m} \Omega \mathrm{cm}$. Their density was $6 \times 10^{10} / \mathrm{cm}^{3}$, and about 4 atomic\% of $\mathrm{P}$ was detected on the plate-like defects by using energy dispersive X-ray spectroscopy (EDX). They suggested that precipitates such as $\mathrm{SiP}$ would form as a result of the super-saturation of $\mathrm{P}$ around $600^{\circ} \mathrm{C}$ during the crystal cooling process. However, in such case, because the $\mathrm{P}$ concentration is low and the crystal structure has not been verified, the exact nature and generation mechanism of defects in crystals heavily doped with $\mathrm{P}$ remain unknown.

Regarding theoretical predictions, to reflect the behavior of voids in crystals heavily doped with As or P, Voronkov et al. ${ }^{25}$ proposed an advanced model based on the formation of complexes between point defects and dopant impurities, i.e., VMs, consisting of a vacancy (V) and a substitutional dopant (Ms), and IMs, consisting of a self-interstitial (I) and a substitutional dopant. Note that since an Ms and interstitial dopant (Mi) can always exchange their positions through a kick-out reaction $(\mathrm{Ms}+\mathrm{I}=\mathrm{Mi})$ via $\mathrm{I}$, they can be regarded as equivalent states. In addition, Nakamura et al. ${ }^{30}$ recently used this model to examine the total point defect concentration, defined as $\mathrm{C}_{\mathrm{VT}}-\mathrm{C}_{\mathrm{IT}}$, as a function of $v / G$ in crystals heavily doped with As or $\mathrm{P}$, where $\mathrm{C}_{\mathrm{VT}}$ is the total vacancy concentration $(\mathrm{V}+\mathrm{VMs})$ and $\mathrm{C}_{\mathrm{IT}}$ is the total self-interstitial concentration (I + IMs or $\mathrm{I}+\mathrm{Mi})$. They showed that above the critical concentration of As or $\mathrm{P}\left(2-3 \times 10^{19}\right.$ atoms $\left./ \mathrm{cm}^{3}\right), \mathrm{C}_{\mathrm{IT}}$ becomes dominant without depending on $v / G$. This is because IMs or $\mathrm{Mi}$, not self-interstitials, become dominant. It was concluded that self-interstitialrelated defects such as dislocations loops and/or stacking faults would not form in $n$-type crystals heavily doped with As or P. However, on wafer production lines, many defects, such as stacking fault tetrahedrons and/or dislocation pairs, are frequently observed in epitaxial layers grown on ntype substrates doped with As or P concentrations much higher than the critical values. Thus, it is speculated that self-interstitial-related defects exist in such heavily doped crystals. The details of the speculation have not yet been clarified.

Crystal manufacturers have continued to improve and develop CZ-Si crystals with much lower resistivity and much higher crystal perfection to meet the demands of advanced power devices. The quest to overcome crystal-quality limitations frequently provides information on unexpected physical phenomena such as new defect formation. Therefore, to control defects in such crystals, we must continue with our experimental and theoretical studies to understand the states of point defects and impurities and their interaction during crystal growth.

\section{SUMMARY}

Abe et al. were the first to propose that the behavior of point defects in growing CZ-Si crystals can be visualized by rapidly cooling after detachment from the Si melt. We applied this method to lightly and heavily B-doped CZ-Si crystals and obtained several new findings, which were reviewed in this paper. Some of the experimental results include previously unpublished ones (Fig. 4a and b). In addition, to examine the behavior of point defects, we performed a two-dimensional pointdefect simulation. Using the one-dimensional point defect distributions calculated along the crystal growth axis, we confirmed that the defect distributions observed in the lightly doped CZ-Si crystals could be reproduced by Voronkov's $v / G$ model, which consists of point defect diffusion and pair recombination.

In lightly B-doped crystals grown at a high pulling rate, excess vacancies in the AOP region aggregate to form voids in a narrow temperature range, $30^{\circ} \mathrm{C}$, below $1100^{\circ} \mathrm{C}$, and this results in a decrease in the vacancy concentration and AOP termination. In the crystals grown with a medium pulling rate, the AOP and OSF-ring regions shrink to the crystal center due to a reduction in the vacancy concentration, and the AOP termination temperature also decreases. Furthermore, in the crystals grown at a low pulling rate, vacancies 
replace self-interstitials as the dominant point defects. As a result, the AOP region shrinks remarkably near the solid-liquid interface, and a self-interstitial dominant region, which is identified to be an interstitial-type dislocation loop region, spreads adjacent to the AOP region across the narrow defect-free region. Furthermore, excess selfinterstitials aggregate at about $1000^{\circ} \mathrm{C}$ to form dislocation clusters, thereby terminating the selfinterstitial dominant region. Furthermore, during growth halting for up to $5 \mathrm{~h}$ after high-speed growth, a dislocation loop region forms and expands in the AOP region through in-diffusion of selfinterstitials from the solid-liquid interface, while excess vacancies in the AOP region diffuse out to the crystal surface and into the bottom of the void region resulting in shrinkage of the $\mathrm{AOP}$ region to an island-like region. The transient behaviors of point defects observed in the lightly B-doped crystals could be reproduced by the point defect simulator.

We also investigated the behavior of point defects in heavily B-doped crystals with different B concentrations grown at high and low pulling rates by employing the rapid cooling method. We found that the point defect behavior in crystals with resistivities as low as $40 \mathrm{~m} \Omega \mathrm{cm}$ is similar to that in lightly B-doped crystals. By reducing the resistivity of the crystals grown with at a high pulling rate, the void and OSF-ring regions began to shrink from $20 \mathrm{~m} \Omega \mathrm{cm}$ and disappeared completely at the crystal center below $10 \mathrm{~m} \Omega \mathrm{cm}$. Such changes in defect regions are very similar to the changes with $v / G$ in lightly doped CZ-Si crystals. As a result, we expected that the crystals with resistivities less than $10 \mathrm{~m} \Omega \mathrm{cm}$ would be self-interstitial dominated. However, we did not observe any self-interstitialrelated defect regions. At present, we cannot judge whether these regions are actually self-interstitial dominated or not. In conclusion, in regard to their application to advanced power devices, the states and behaviors of point defects in heavily doped crystals with various impurities remain to be elucidated.

\section{CONFLICT OF INTEREST}

The authors declare that they have no conflict of interest.

\section{OPEN ACCESS}

This article is licensed under a Creative Commons Attribution 4.0 International License, which permits use, sharing, adaptation, distribution and reproduction in any medium or format, as long as you give appropriate credit to the original author(s) and the source, provide a link to the Creative Commons licence, and indicate if changes were made. The images or other third party material in this article are included in the article's Creative Commons licence, unless indicated otherwise in a credit line to the material. If material is not included in the article's Creative Commons licence and your intended use is not permitted by statutory regulation or exceeds the permitted use, you will need to obtain permission directly from the copyright holder. To view a copy of this licence, visit http://creativecom mons.org/licenses/by/4.0/.

\section{APPENDIX: POINT DEFECT SIMULATION}

The model equations are Eqs. (1) and (2), ${ }^{9,10}$ where $C_{\mathrm{v}}$ and $C_{\mathrm{i}}$ are the actual concentrations of vacancies and self-interstitials (the superscript $*$ denotes values under equilibrium conditions) and $D_{\mathrm{v}}$ and $D_{\mathrm{i}}$ are the diffusion constants of vacancies and self-interstitials. The first term on the right side of each equation denotes the diffusion effect due to the concentration gradients of the point defects, and the second term denotes the effect of crystal growth on the point defect concentrations, where $V_{\mathrm{p}}$ is the crystal growth rate and $Z$ is the growth direction coordinate. The third term represents the recombination reaction between vacancies and self-interstitials, where $k_{\text {iv }}$ is a reaction constant called the Frenkel recombination coefficient. Since the cooling rate of the crystal during growth is slow enough compared with the pair recombination rate at high temperatures near the melting point, it was assumed that the mass action law for pair recombination expressed in Eq. (5) is satisfied in a sufficiently short time. ${ }^{31}$ In addition, we assumed the following boundary conditions:

(1) The concentrations of vacancies and self-interstitials at the solid-liquid interface are the thermal equilibrium concentrations at the melting point.

(2) The concentrations of vacancies and self-interstitials on the surface of the crystal are the thermal equilibrium concentrations at that temperature.

The diffusion constants of $D_{\mathrm{v}}$ and $D_{\mathrm{i}}$ are expressed in Eq. (3) by using the diffusion activation energies of $E_{\mathrm{dv}}$ for vacancies and $E_{\mathrm{di}}$ for self-interstitials and the Boltzmann constant $k_{\mathrm{B}}$. The thermal equilibrium concentrations of $C_{\mathrm{v}}^{*}$ and $C_{\mathrm{i}}^{*}$ are expressed in Eq. (4) by using the formation energies of $E_{\mathrm{fv}}$ for vacancies and $E_{\mathrm{fi}}$ for self-interstitials. The subscript $\mathrm{m}$ means the melting point.

$$
\begin{gathered}
\frac{\partial C_{\mathrm{v}}}{\partial t}=\nabla\left(D_{\mathrm{v}} \nabla C_{\mathrm{v}}\right)-V_{\mathrm{p}} \frac{\partial C_{\mathrm{v}}}{\partial Z}-k_{\mathrm{iv}}(T)\left(C_{\mathrm{i}} C_{\mathrm{v}}-C_{\mathrm{i}}^{*} C_{\mathrm{v}}^{*}\right) \\
\frac{\partial C_{\mathrm{i}}}{\partial t}=\nabla\left(D_{\mathrm{i}} \nabla C_{\mathrm{i}}\right)-V_{\mathrm{p}} \frac{\partial C_{\mathrm{i}}}{\partial Z}-k_{\mathrm{iv}}(T)\left(C_{\mathrm{i}} C_{\mathrm{v}}-C_{\mathrm{i}}^{*} C_{\mathrm{v}}^{*}\right)
\end{gathered}
$$


Table I. Physical parameters of point defects in silicon derived using heuristic methods ${ }^{9,10}$

\begin{tabular}{|c|c|c|c|c|c|c|c|}
\hline \multicolumn{4}{|l|}{ Vacancy } & \multicolumn{4}{|c|}{ Self-interstitial } \\
\hline$D_{\mathrm{vm}}\left(\mathrm{cm}^{2} / \mathrm{s}\right)$ & $E_{\mathrm{dv}}(\mathrm{eV})$ & $C_{\mathrm{vm}}^{\mathrm{eq}}\left(\mathrm{cm}^{-3}\right)$ & $E_{\text {fv }}(\mathbf{e V})$ & $D_{\mathrm{im}}\left(\mathrm{cm}^{2} / \mathbf{s}\right)$ & $E_{\text {di }}(\mathbf{e V})$ & $\mathrm{C}_{\mathrm{im}}^{\mathrm{eq}}\left(\mathrm{cm}^{-3}\right)$ & $E_{\mathrm{fi}}(\mathrm{eV})$ \\
\hline $6.310 \times 10^{-5}$ & 0.40 & $4.580 \times 10^{14}$ & 3.84 & $7.656 \times 10^{-4}$ & 1.18 & $3.174 \times 10^{14}$ & 3.77 \\
\hline
\end{tabular}

$$
\begin{gathered}
D_{\mathrm{v}}=D_{\mathrm{vm}} \exp \left(-\frac{E_{\mathrm{dv}}}{k_{\mathrm{B}}}\left(\frac{1}{T}-\frac{1}{1685}\right)\right), \\
D_{\mathrm{i}}=D_{\mathrm{im}} \exp \left(-\frac{E_{\mathrm{di}}}{k_{\mathrm{B}}}\left(\frac{1}{T}-\frac{1}{1685}\right)\right) \\
C_{\mathrm{v}}^{*}=C_{\mathrm{vm}}^{*} \exp \left(-\frac{E_{\mathrm{fv}}}{k_{\mathrm{B}}}\left(\frac{1}{T}-\frac{1}{1685}\right)\right), \\
C_{\mathrm{i}}^{*}=C_{\mathrm{im}}^{*} \exp \left(-\frac{E_{\mathrm{fi}}}{k_{\mathrm{B}}}\left(\frac{1}{T}-\frac{1}{1685}\right)\right) \\
C_{\mathrm{i}} C_{\mathrm{v}}=C_{\mathrm{i}}^{*} C_{\mathrm{v}}^{*}
\end{gathered}
$$

This simulator uses the physical parameters of the equilibrium concentrations and diffusion rates of vacancies and self-interstitials that were optimized by Nishimoto, ${ }^{9,10}$ who used a generic algorithm method $^{32}$ and simulated annealing ${ }^{33}$; therefore, the V-I boundary, where vacancies and self-interstitials are in balance in the growing CZ-Si crystals, are reproduced as accurately as possible. Table I shows the physical parameters of point defects optimized by Nishimoto. ${ }^{9,10}$

In addition, the temperature in the crystal and the shape of the solid-liquid interface during growth were calculated using commercially available software, FEMAG, ${ }^{34}$ which is a simulator based on a two-dimensional axisymmetric model for global heat transfer and Si melt flow. The thermophysical properties of the CZ furnace components and the turbulent Prandtl number for the Si melt flow were also optimized by Nishimoto ${ }^{9}$ so that the measured temperature distribution in the crystal and the shape of the solid-liquid interface were reproduced as accurately as possible.

\section{REFERENCES}

1. M. Kato, Y. Ikeda, and Y. Kitagawara, Jpn. J. Appl. Phys. 35, 5597 (1996).

2. T. Ueki, M. Itsumi, and T. Takeda, Appl. Phys. Lett. 70, 1248 (1997).

3. S. Sadamitsu, S. Umeno, Y. Koike, M. Hourai, S. Sumita, and T. Shigematsu, Jpn. J. Appl. Phys. 32, 3675 (1993).

4. H. Harada, T. Abe, and J. Chikawa, in Semiconductor Silicon 1986, ed. H. R. Huff, T. Abe, and B. O. Kolbesen (Electrochemical Society, Pennington, 1986), p. 76.

5. T. Abe and H. Takeno, in Materials Research Society Symposium Proceedings, vol. 3, (1992), p. 51.

6. M. Hourai, T. Nagashima, E. Kajita, S. Miki, T. Shigematsu, and M. Okui, J. Electrochem. Soc. 142, 3193 (1995).
7. M. Okui, T. Tanaka, T. Kanda, and T. Ono, Oyo Buturi 66, 707 (1997)

8. M. Okui, T. Tanaka, T. Kanda, T. Ono, K. Egashira, and S. Kuragaki, in Proceedings of the Kazusa Akademia Park Forum on the Science and Technology of Silicon Materials (1997), pp. 444-445.

9. M. Nishimoto, Doctoral Thesis, (Kyushu University, Japan, 2009).

10. M. Nishimoto, K. Nakamura, M. Hourai, T. Ono, W. Sugimura, and T. Motooka, J. Jpn. Inst. Met. Mater. 75, 657 (2011).

11. V.V. Voronkov, J. Cryst. Growth 59, 625 (1982).

12. H. Nishikawa, T. Tanaka, Y. Yanase, M. Hourai, M. Sano, and H. Tsuya, Jpn. J. Appl. Phys. 36, 6595 (1997).

13. D. Guidotti, J.S. Batchelder, and M.A. Taubenblatt, Appl. Phys. A 55, 139 (1992).

14. J. Ryuta, E. Morita, T. Tanaka, and Y. Shimanuki, Jpn. J. Appl. Phys. 29, L1947 (1990).

15. E. Asayama, T. Ono, T. Tanaka, H. Nishikawa, T. Nomachi, and M. Hourai, J. Jpn. Assoc. Cryst. Growth 26, 258 (1999).

16. M. Suhren, D. Graf, U. Lambert, and P. Wagner, High Purity Silicon IV, Vol. 96-13, ed. C.L. Claeys, P. RaiChoudhury, P. Stalhofer, and J.E. Maurits (Pennington: Electrochemical Society, 1996), p. 132.

17. M. Hourai, E. Kajita, T. Nagashima, H. Fujiwara, S. Umeno, S. Sadamitsu, S. Miki, and T. Shigematsu, Materials Science Forum, Vol. 196-201 (Stafa: Trans Tech Publications, 1995), p. 1713.

18. M. Hourai, T. Nagashima, H. Nishikawa, W. Sugimura, T. Ono, and S. Umeno, Phys. Status Solidi A 216, 1800664 (2019). https://doi.org/10.1002/pssa.201800664.

19. A.J.R. de Kock, W.T. Stacy, and W.M. van de Wijgert, Appl. Phys. Lett. 34, 611 (1979).

20. W.R. Thurber, R.L. Mattis, Y.M. Liu, and J.J. Filliben, J. Electrochem. Soc. 127, 2291 (1980).

21. E. Dornberger, D. Graf, M. Suhren, U. Lambert, P. Wagner, F. Dupret, and W. von Ammon, J. Cryst. Growth 180, 343 (1997).

22. W.V. Ammon, in Proceedings of 2nd International Symposium on Advanced Science and Technology of Silicon Materials, ed. M. Umeno, vol. 233, (1996).

23. K. Sueoka, E. Kamiyama, and J. Vahellemont, J. Appl. Phys. 114, 153510 (2013).

24. K. Nakamura, R. Suewaka, T. Saishoji, and J. Tomioka, in The 4th Forum on the Science and Technology of Silicon Materials, ed. H. Yamada-Kaneta and K. Sumino (The Japan Technical Information Service, Tokyo, 2003), p. 161.

25. V. Voronkov, R. Falster, M. Porrini, and J. Duchini, Phys. Status Solidi A 209, 1898 (2012).

26. G. Borionetti, D. Gambaro, M. Porrini, and V.V. Voronkov, ECS Proc. 2002, 505 (2002).

27. W. Sugimura, T. Ono, S. Umeno, M. Hourai, and K. Sueoka, ECS Trans. 2, 95 (2006).

28. M. Porrini, J. Duchin, and A. Bazzali, Cryst. Res. Technol. 49, 564 (2014). https://doi.org/10.1002/crat.201300392.

29. T. Senda, T. Ishikawa, H. Fujimori, H. Matsumura, S. Narimatsu, Y. Abe and T. Horikawa, in Extended Abstracts (The 78th JSAP Autum Meeting, 2017), 7p-PB6-5 (in Japanese).

30. K. Nakamura, S. Narimatsu, T. Senda, S. Maeda, in Extended Abstracts (The 80th JSAP Autumn Meeting, 2019), 18a-C212-5 (in Japanese). 
31. K. Nakamura, T. Saishoji and J. Tomioka, in High Purity Silicon V, ed. C. L. Claeys et al., (1998) PV98 13, p. 41.

32. J.H. Holland, Adaptation in Natural and Artificial Systems (Ann Arbor: University of Michigan Press, 1975).

33. S. Kirkpatrik, C.D. Galett, and M.P. Vecchi, Science 220, 671 (1983).
34. F. Dupret, P. Nicodeme, Y. Ryckmans, P. Wouters, and M. Crochet, Int. J. Heat Mass Transf. 33, 1849 (1990).

Publisher's Note Springer Nature remains neutral with regard to jurisdictional claims in published maps and institutional affiliations. 